\title{
Neonatal onset of hot water reflex seizures in monozygotic twins subsequently manifesting episodes of alternating hemiplegia
}

\section{Gemma Incorpora, Piero Pavone, Martino Ruggieri*, Mariadonatella Cocuzza, Luigi Mazzone, Enrico Parano, Michael Privitera}

\author{
Department of Paediatrics, University of Catania, Italy
}

Received 12 January 2007; received in revised form 16 May 2007; accepted 14 August 2007

Available online 21 December 2007

\section{KEYWORDS}

Reflex epilepsy;

Genetic disease;

EEG

\begin{abstract}
Summary We report on monozygotic twins with neonatal onset of daily reflex seizures triggered by hot water. Video record during the hot water bathing showed clinical signs consistent with a reflex seizure. The numbers of episodes were markedly reduced when the mother began bathing the children with reduced temperature bath water. At the age of 20 months, the twins developed episodes of paroxysmal disturbances including alternating hemiplegia. These two patients are the youngest reported cases of reflex hot water seizures, and the only reported cases in which reflex hot water seizures subsequently manifested episodes of alternating hemiplegia.

(c) 2007 Elsevier B.V. All rights reserved.
\end{abstract}

\section{Introduction}

We report the clinical and neurophysiologic findings and outcome data of two 5-year-old monozygotic twins who presented with episodes of hot water reflex seizures from their first days of life followed, by the age of 20 months, by clinical episodes of cerebral dysfunction including alternating hemiplegia $(\mathrm{AH})$.

\footnotetext{
* Corresponding author at: ISN, National Research Council, Department of Paediatrics, University of Catania, Viale R. Margherita, 6, 95123 Catania, Italy. Tel.: +39 0953782487; fax: +39095 551582 .

E-mail address: m.ruggieri@isn.cnr.it (M. Ruggieri).
}

\section{Clinical reports}

\section{Twin 1}

This 5-year-old girl was born to unrelated, healthy, Italian parents after a full-term pregnancy. She was delivered by caesarean section after a normal pregnancy. Her birth weight was $2.650 \mathrm{~g}$, height was $50 \mathrm{~cm}$ and head circumference was $35 \mathrm{~cm}$. Her psychomotor development was scored as borderline as she started walking and vocalising at 16 months of age.

The parents recalled that their daughter experienced daily paroxysmal attacks at the same time as daily hot water baths since the age of 3 days. These attacks occurred only during immersion in a hot bath or when hot water was sprin- 


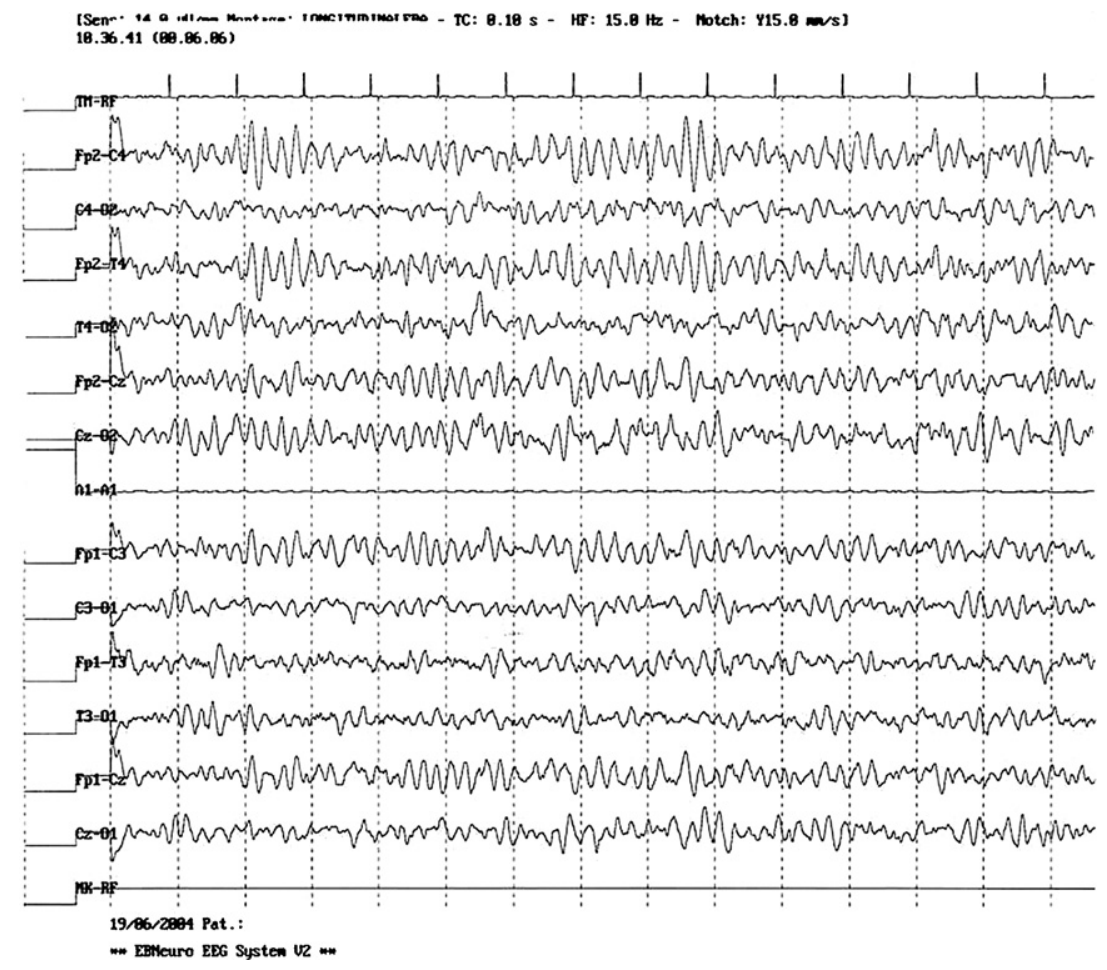

Fig. 1 Case 1: EEG recording at age 25 months showing a normal slow background activity.

kled over the body; they did not occur spontaneously. The bath water temperature was kept around $30^{\circ} \mathrm{C}$. Clinically, the attacks were characterized by irritability and an unusual and unprovoked smile followed by a fixed stare with head deviation and upper-limb hypertonia to one side, guttural sounds and noisy breathing. The episodes lasted from a few seconds to 3-4 min, and usually ceased with a cry while the mother was drying the child. Generalized hypotonia, which was more evident on one side (usually opposite to the side of head deviation), followed each episode; thereafter, the girl fell asleep for several hours. Both parents reported that the episodes had similar clinical features, although they varied in intensity, and that an episode occurred each time the child had a hot bath. Bathing on the beach during the summer did not provoke an attack.

At the age of 24 months she had an episode characterised by confusion, followed by generalised hypotonia, ataxia, right hemiplegia, impaired speech and strabismus lasting 3 days. She was referred to an hospital elsewhere in our region where she was diagnosed as having acute encephalopathy. At that time cerebrospinal fluid analysis yielded normal results.

When first referred to our University Department of Paediatrics at the age of 25 months this twin's general conditions were good. Her height, weight and head circumference were measured at the 50th percentile. There were neither dysmorphic features nor cutaneous birth marks or lesions. General examination was normal. Neurological examination showed that the girl was able to walk unassisted. The language was composed of few disyllable words. Routine metabolic, immunologic, and infective blood and urinalysis gave normal results. Full ophthalmologic exami- nation was normal. Brain magnetic resonance imaging (MRI), MRI angiography and positron emission tomography (PET) scans were normal. Psychological testing by means of the CAT/CLAMS tool gave the following results: CAT $=80$ and CLAMS $=80$.

The first interictal electroencephalogram (EEG) showed normal slow background activity in the absence of definite spikes or spikes and waves (Fig. 1). To record an ictal EEG in this child during immersion in hot water, with the consent of the parents, we recreated similar conditions to those at home and used simultaneous EEG and video recording. On video recording a few minutes after her body was immersed in the hot water the girl had a paroxysmal episode characterized by laughter and a tonic fixation and deviation of the eyes accompanied by movements of the head to the left side with tonic flexion of the left arm, speech arrest, noisy breathing, purposeless chewing and salivation. EEG recording along with recording artefacts showed generalised high voltage sharp waves and high-amplitude spikes that were independent in the right frontal and left posterior area (Fig. $2 a$ and $b$ ). The recorded episode was followed by generalized hypotonia lasting a few minutes, and the girl then fell asleep for $1 \mathrm{~h}$. The magnetic resonance imaging (MRI) scan was normal.

Thereafter, the parents were advised to use lower water temperatures (e.g., between 25 and $28^{\circ} \mathrm{C}$ ) when bathing the girl and to use the shower instead of full immersion in a bath. With these simple procedures the parents observed cessation of the seizures.

Four months later, the mother reported that the child had two new episodes: the first was during a shower and the second during a shampooing, both of which were performed at 

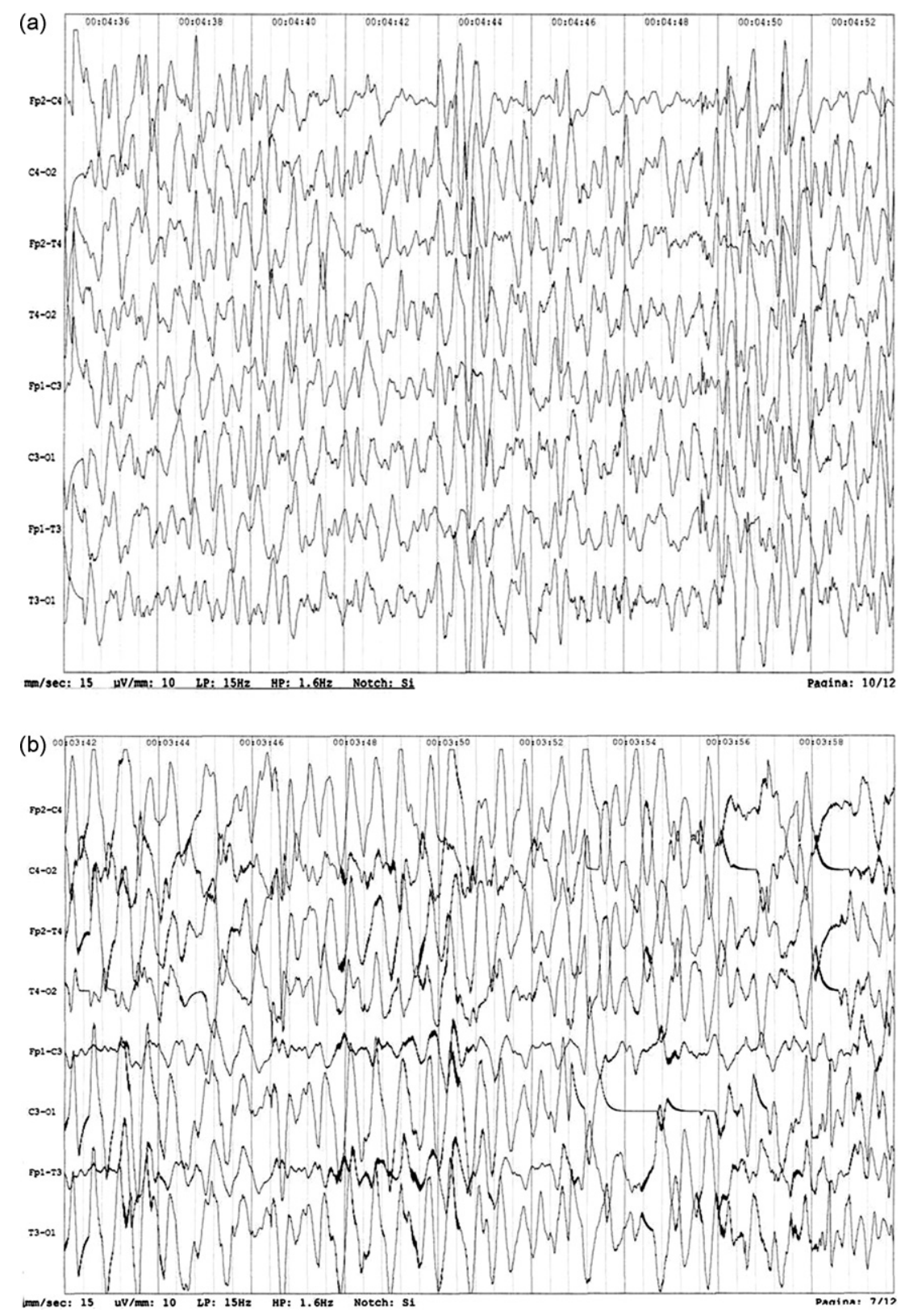

Fig. 2 Case 1: EEG recording at age 25 months during hot water bathing shows (a and b) generalised high voltage sharp waves and high-amplitude spikes that were independent in the frontal and posterior area.

the recommended lower temperatures. In addition, the girl experienced a convulsion which lasted $10 \mathrm{~min}$ and occurred during a febrile episode. A repeated interictal EEG showed normal background activity with paroxysmal generalized slow waves (Fig. 3). It was decided not to start any treatment.

At age 2 years and 6 months, the girl had an episode, likely similar to that experienced at age 24 months, which was unrelated to hot water immersion and was characterised by confusion, ataxia, salivation, autonomic disturbances, speech arrest, right hemiplegia and inconstant tonic deviation of the eyes. This episode lasted almost an entire day. These signs were all seen within one type of episode and ceased during sleep. The ictal EEG recorded during this episode showed slow generalized high-amplitude activity that was more evident in the frontal-central region without definite spikes or sharp waves (Fig. 4). The EEG recordings performed a few days after the episode were normal (Fig. 5). The girl was given topiramate $5 \mathrm{mg} / \mathrm{kg}$ per day.

During her third year of life the girl continued to suffer similar episodes to the last recorded attacks. These attacks, which were about 20, lasted hours to days and were characterized by confusion, lethargy, ataxia, salivation, speech arrest, hemiplegia, generalised hypotonia and contralateral tonic deviation of the eyes. These signs were all seen within one type of episode and ceased during sleep. The hemiplegia occurred on the right and only twice on the 


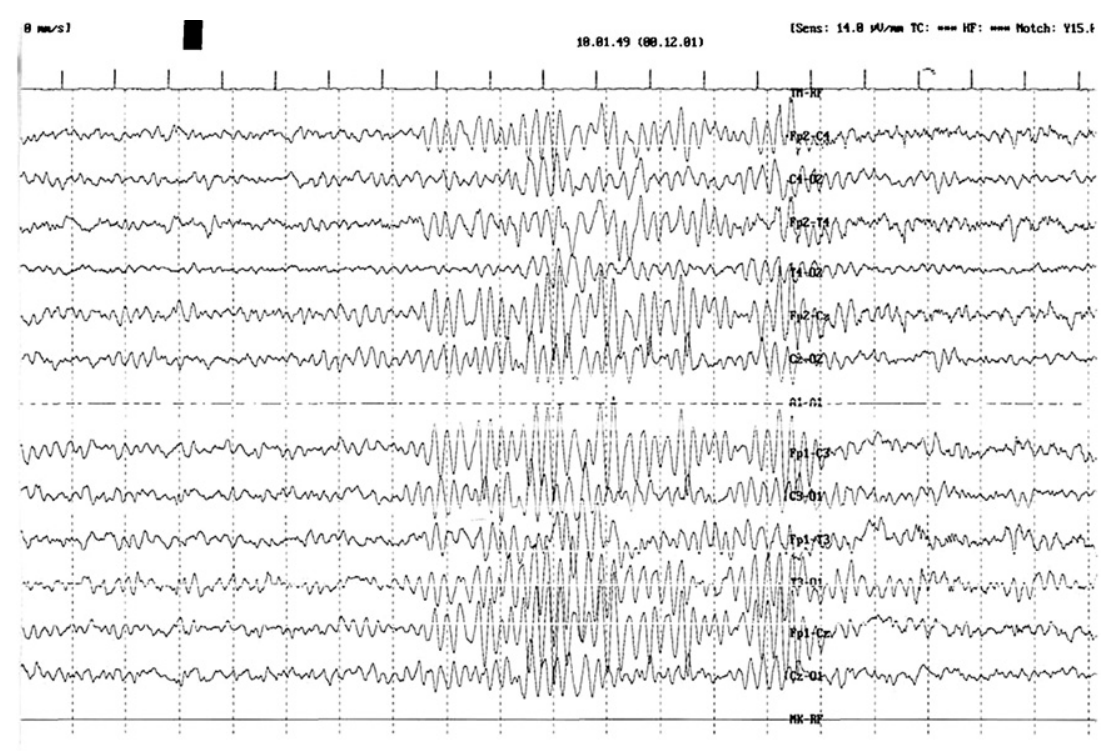

-. Eamaro Evi Susten v2 -.

Fig. 3 Case 1: Interictal EEG obtained at age 30 months showed normal background activity with sporadic paroxysmal generalised slow waves.

left. During one of such episodes holter EEG record showed normal sleep activity (Fig. 6). Repeated EEG recordings showed generalised slow background activity without focal anomalies.

From the age of 4 years to the present age, this girl has had in addition to the long lasting episodes previously reported single, occasional, brief episodes of mild hemiplegia, eye deviation and attacks of dystonia of the hands. Slowing of movements, motor and gait uncoordination and unintelligible speech are constantly present. Currently, she is still under topiramate, attending primary school and undergoing speech and rehabilitation therapy.

\section{Twin 2}

The past medical and current clinical history of this patient is similar to that of her twin: all episodes occurred at the same time as her sister. In summary, she had mild developmental delay and attacks precipitated by hot water baths from the first days of life.

Similarly to her sister, at the age of 22 months, she was hospitalised with signs and symptoms of an acute encephalopathy even though to a lesser degree to her sister.

She first came to our observation with her sister (i.e., at the age of 25 months) and her attacks ceased (as did those of her sister) when the parents reduced the water temperature during baths.

At age 2 years and 6 months she began to suffer paroxysmal attacks that were not associated with hot water immersion and/or hot water sprinkling and were characterised by confusion, lethargy, ataxia, salivation, autonomic disturbances, speech arrest, (exclusively) right hemiplegia, and generalised hypotonia. These signs were all seen within one type of episode and ceased during sleep. Full metabolic, immunologic and infective blood and urinalysis showed normal results and brain MRI and MRI angiography scans were normal. The ictal and interictal EEGs were identical to those of her sister. She is currently receiving topiramate $5 \mathrm{mg} / \mathrm{kg}$ per day. The mother recalled that, compared with those of her twin sister, all the attacks of hot water seizures were less severe in intensity or duration whilst the attacks of hemiplegia more severe.

In both twins, a full search for mutations in the ATP1A2 gene was negative.

\section{Discussion}

These twins showed very unusual clinical features and disease course. At their first referral to our Department, at the age of 25 months, the episodes were induced by hot water immersion and we were able to document the attacks after immersion in a bathtub filled with hot water. Video recording and EEG during hot water immersion showed a clinical seizure with eye deviation and tonic arm flexion that lasted approximately $1 \mathrm{~min}$. Simultaneous EEG showed high-voltage sharp waves and high amplitude spikes that were independent in the right frontal and left posterior area. Clinically, the attacks reflected what was reported by parents since the twins' first days of life. The diagnosis was delayed as the condition was not fully recognised by the general paediatrician and by the parents themselves, who considered the episodes a non-hazardous and normal reaction to hot water. The frequency of the episodes was markedly reduced when the parents started bathing both twins at lower water temperatures.

Hot water reflex seizures, according to the International League Against Epilepsy (ILAE), are included in the group of reflex epilepsies. This condition is more frequent in some 
(a)

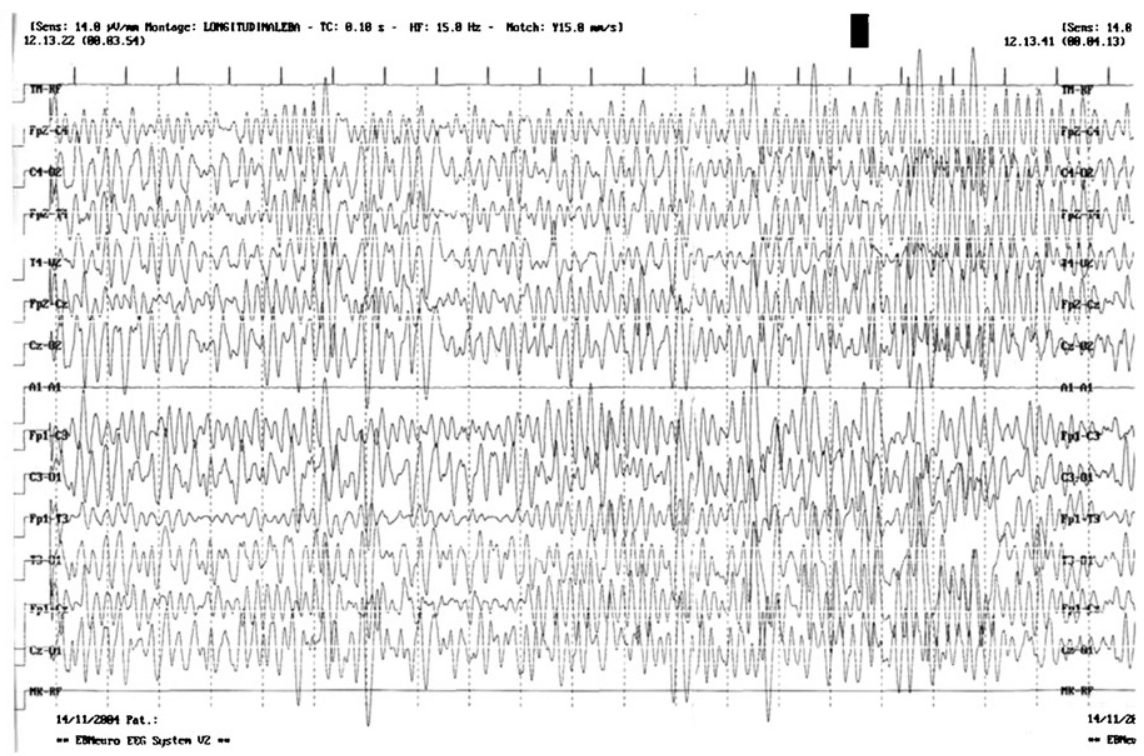

(b)

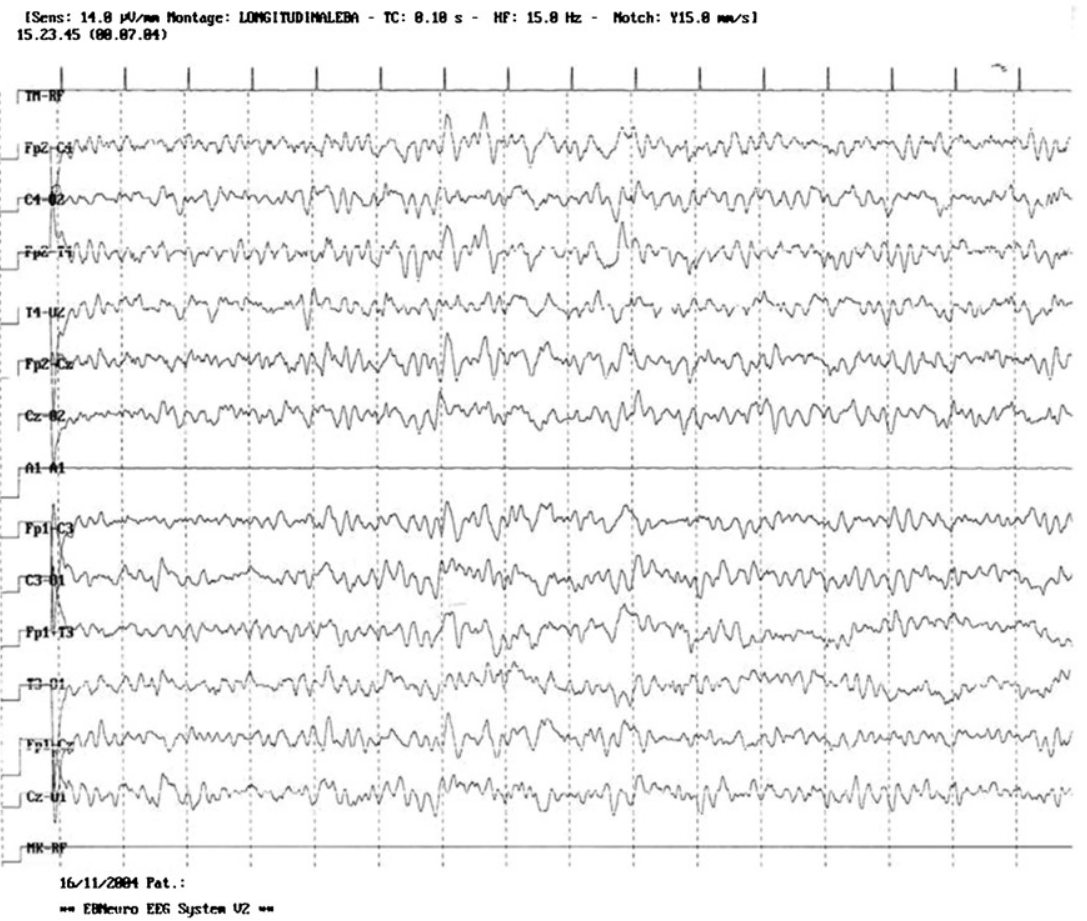

Fig. 4 Case 1: Ictal EEG recorded at age 32 months shows slow generalised high-amplitude activity more evident in the frontalcentral region without definite spikes or sharp waves.

countries, including India and Turkey, but is uncommon in Europe particularly in Italy, where it has been recorded only rarely (Satischandra, 2003). As reflex epilepsy occurs with other sensory activities such as reading, eating and listening or with sensory stimuli such as flickering lights and colour patterns, hot water immersion may trigger clinical events that can be classified as seizures. The pathophysiology of reflex seizures is unknown and, in many cases, its relationship with epilepsy has not yet been demonstrated. In the most recent ILAE classification of epilepsy syndromes (Engel, 2001) "reflex seizures"' are categorised as a con- ditions involving epileptic seizures that do not require a diagnosis of epilepsy. On the other hand, hot water reflex epilepsy, similar to many other types of reflex epilepsy, is generally benign and self-limited. Hot water epilepsy has been reported in cases of generalised or focal epilepsy. Yalcin et al. (2006) published a large series of cases with hot water epilepsy in which 20 of 25 subjects had complex partial seizures, whereas others had primary generalised seizures.

At the age of 30 months, both twins began to experience numerous, spontaneous episodes of neurological 


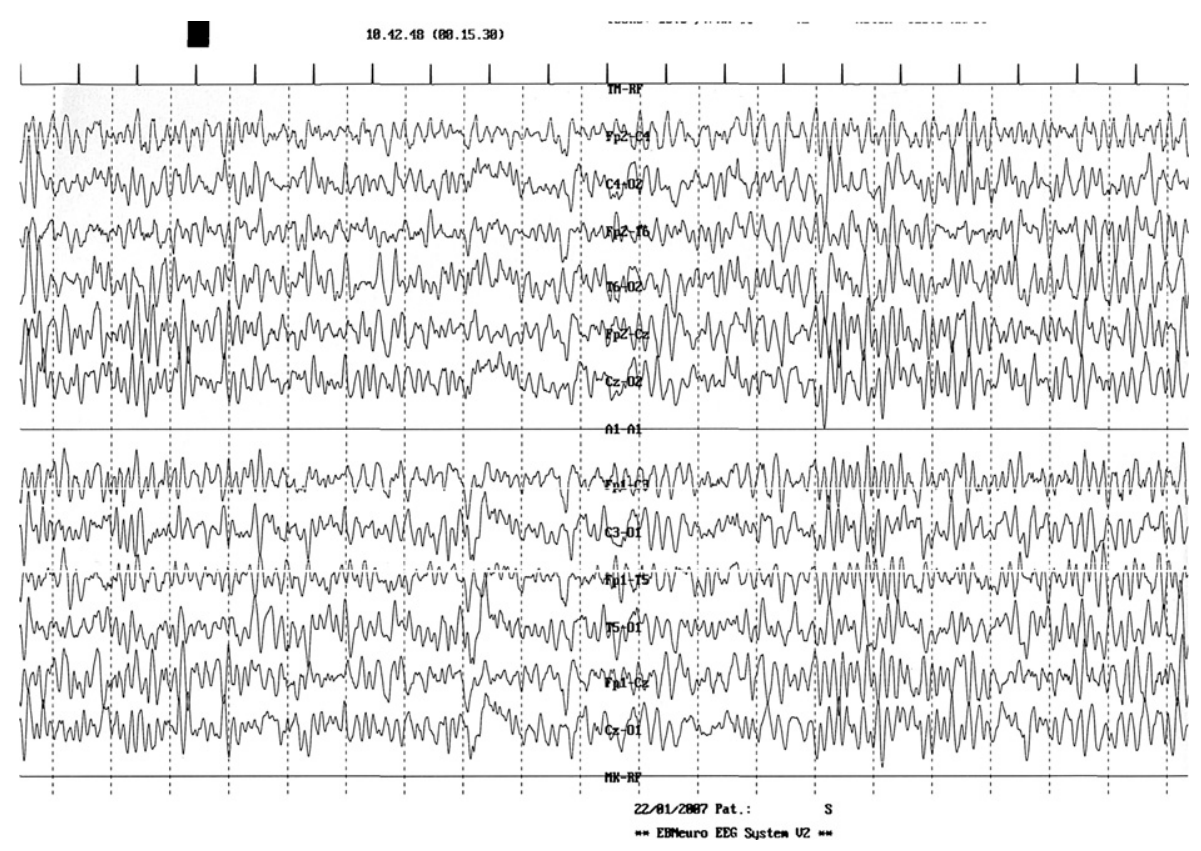

Fig. 5 Case 1: EEG recording obtained 2 days after the EEG shown in Fig. 4 reveals marked reduction of previous findings.

dysfunction characterised by dystonic movements, ataxia, eye deviation, autonomic disturbances, and hemiplegia. All these signs and symptoms occurred mainly within one type of episode and ceased during sleep. The hemiplegic attacks affected prevalently the right side and never occurred on both sides at the same time. These second type of attacks could be related to one of these conditions:

(1) complex partial seizures with subsequent Todd's paralysis;

(2) alternating hemiplegia of childhood (classical or atypical form);

(3) a new distinct entity.

Complex partial seizures could be excluded on the basis of the clinical aspects of episodes and the repeated normal EEG ictal and interictal recordings.

Alternating hemiplegia of childhood according to the criteria suggested by Aicardi (Aicardi, 1987; Bourgeois et al., 1993) is characterised by: (1) onset before 18 months of age; (2) repeated attacks of hemiplegia involving either side of the body; (3) other paroxysmal disturbances including tonic or dystonic spells, nystagmus or other oculomotor disturbances, dyspnoea, and various autonomic phenomena, occurred during hemiplegic bouts or in isolation; (4) episodes of bilateral hemiplegia observed either during attacks that started unilaterally at the time hemiplegia shifted to the contralateral side or from the beginning of attacks; (5) sleep consistently produced immediate disappearance of symptoms which reappeared $10-20$ min children awakened during long lasting boots; (6) developmental delay, mental retardation and neurological abnormalities including choreoathetosis, or ataxia. Our cases did not fully satisfied these crite- ria (Aicardi, 1987; Bourgeois et al., 1993). In particular, the attacks likely started at age 22 months (presumably with the supposed attack of acute encephalopathy); the twins never experienced double hemiplegia; the ictal and interictal EEG showed slow background activity without focal abnormalities which are regarded by most investigators part of the spectrum of $\mathrm{AH}$; in addition (even though not so specific) the DNA analysis for the ATP1A2 gene (Bassi et al., 2004) was negative in either twin. Notably, AH starting with or as hot water reflex seizures has never been previously reported even though Mikati et al. (2000) report that hot water and hot bath are between the provoking factor of $\mathrm{AH}$ in almost $35 \%$ of cases.

What is the relationship between the hot water reflex episodes suffered by these patients in the first 2 years of life, and the subsequent episodes that were unrelated to hot water? We think that the episodes of alternating hemiplegia did not imply that these cases had alternating hemiplegia of childhood but it appears likely that these twins had either an atypical form of alternating hemiplegia (starting as hot water seizures) or a condition showing similarities to alternating hemiplegia but actually configuring a distinct entity.

We reported these case studies as we think they could be important for several reasons: (1) hot water reflex attacks are extremely rare in our geographical area (i.e., Europe) and in our region (Sicily); (2) to the best of our knowledge, the occurrence of hot water seizures in twins has not been previously reported; (3) the clinical presentation with a very early onset, and the subsequent development of clinical neurological dysfunction including attacks of hemiplegia make the clinical features of our patients very unusual and probably unique. 
(a)

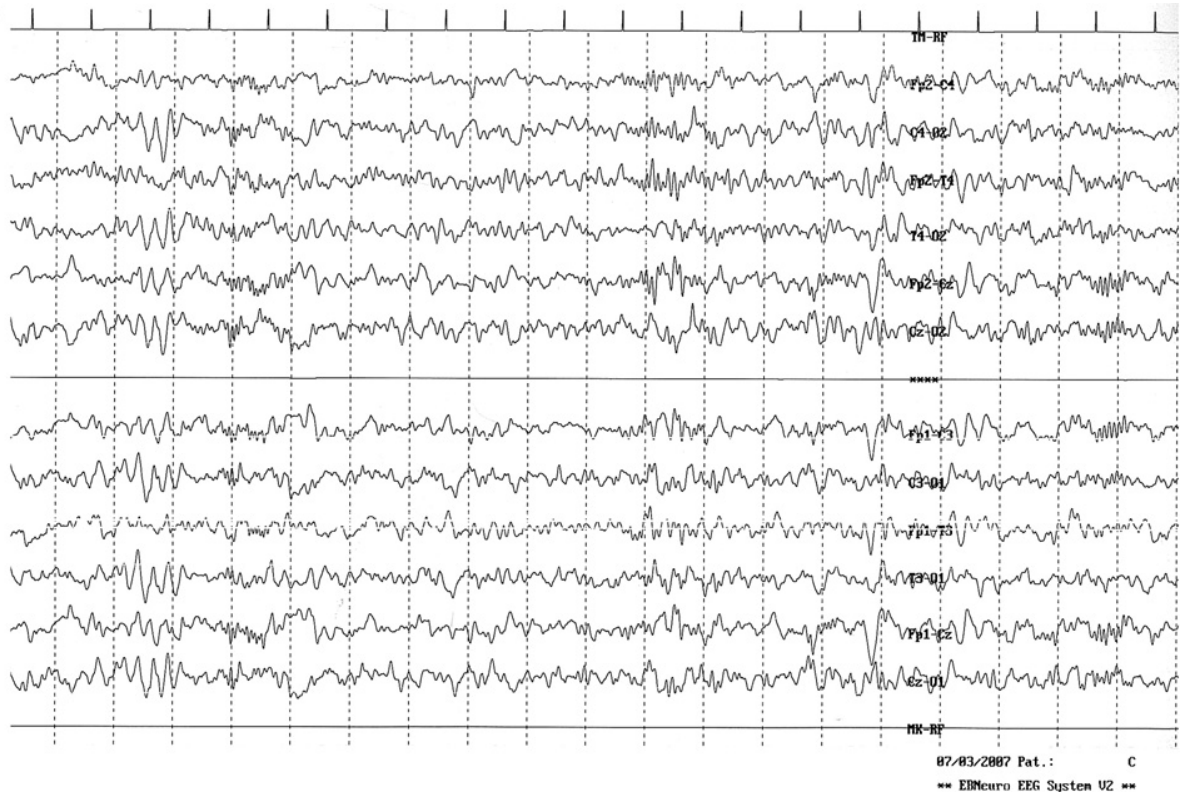

(b)

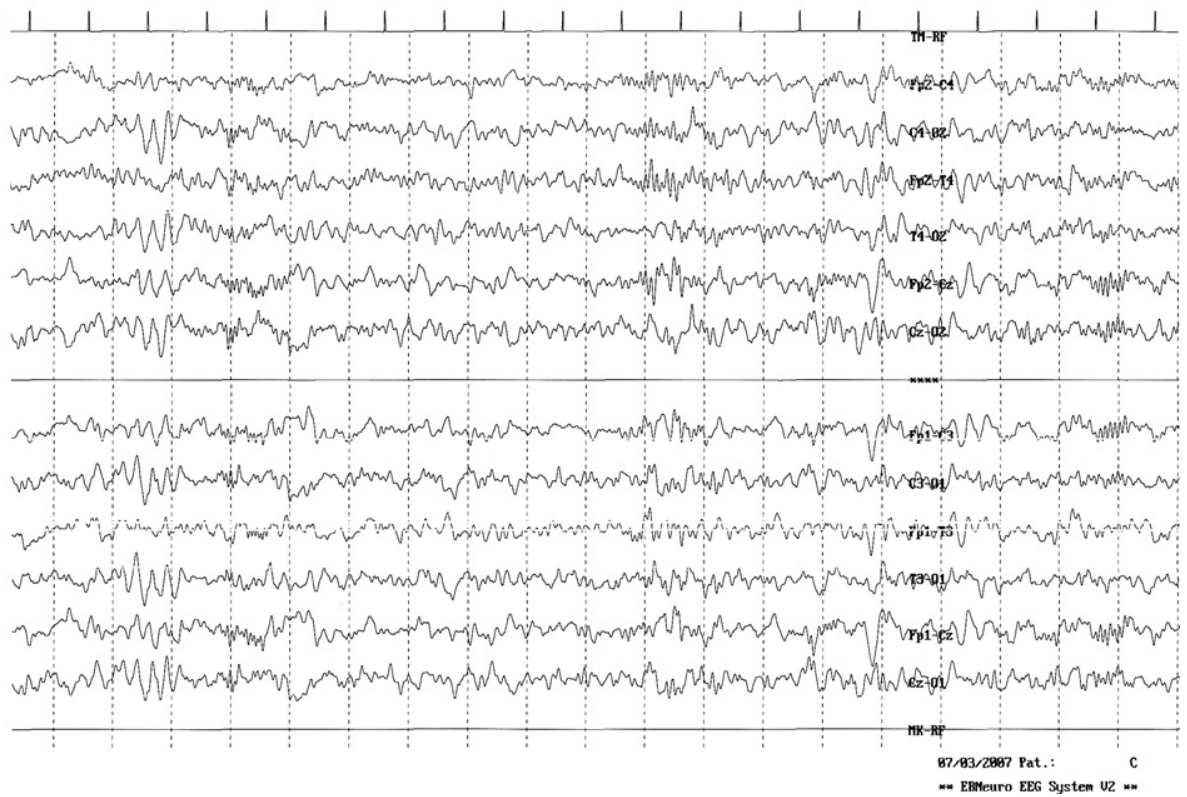

Fig. 6 Holter EEG (a) and standard EEG (b) recorded during the hemiplegic attack, at age 3 years, showed slow activity on awake without focal anomalies and normal sleep activity.

\section{References}

Aicardi, J., 1987. Alternating hemiplegia of childhood. Int. Pediatr. 2, 115-119.

Bassi, M.T., Bresolin, N., Tonelli, A., Nazos, K., Crippa, F., Baschirotto, C., Zucca, C., Bersano, A., Dolcetta, D., Boneschi, F.M., Barone, V., Casari, G., 2004. A novel mutation in the ATP1A2 gene causes alternating hemiplegia of childhood. J. Med. Genet. $41,621-628$.

Bourgeois, M., Aicardi, J., Goutieres, F., 1993. Alternating hemiplegia of childhood. J. Pediatr., 673-679.
Engel, J., 2001. Proposed diagnostic scheme for epileptic seizures and epilepsy. Epilepsia 42, 796-803.

Mikati, M.A., Kramer, U., Zupanc, M.L., Shanahan, R.J., 2000. Alternating hemiplegia of childhood: clinical manifestations and long-term outcome. Pediatr. Neurol. 23, 134-141.

Satischandra, P., 2003. Geographically specific epilepsy syndromes in India, hot-water epilepsy. Epilepsia 44 (Suppl. 1), 29-32.

Yalcin, A.D., Toydem, R.H.E., Fort, A.H., 2006. Hot water epilepsy, clinical and electroencephalographic features in 25 cases. Epilepsy Behav. 9 (1), 89-94. 